trolled by gravitational crystal settling, as he accepts it, is independent of a pre-existing floor slope. Some pertinent, recent publications on the Ardnamurchan cone sheets and the Skye hybrid (marscoite) bodies are not referred to. Early tidying-up is a natural requisite for an hypothesis that will need severe testing.

The mechanics of emplacement is a local problem, whereas the genesis of the British Tertiary magmas relates to universal problems. Work over the past decade or so has concentrated on the latter, and yet the origin of the granitic magmas remains in dispute. Most petrological, experimental and isotopic studies favour an origin by remelting of the crustal rocks (anatexis) rather than by crystal fractionation of the basaltic magmas. Walker's model leaves little doubt that only crustal remelting is acceptable, yet surprisingly he does not refer to that clue or to magma genesis in general. His granitic diapirs are shown as developing before the underlying cylinder of basic intrusives has crystallised, which would be impossible if they were late fractions of a solidifying basic intrusive, quite apart from the fact that only a very small volume of granite is produced by the latter process. Proponents of the fractionation hypothesis will not approve of Walker's granitic diapirs, which makes it all the more important that his hypothesis be explored to the full, and consideration be given to the several broader petrogenetic implications.

\section{Silicon wafers without sawing}

from Andrew Holmes-Siedle

$\mathrm{O}_{\mathrm{NE}}$ annoying deficiency in semiconductor device technology today is the limitation in size of the singlecrystal ingots of semiconductors (such as silicon) which can be grown. Nearly all mass-produced ingots are grown by slow pulling from the melt (the Czochralski method). The best ingots that can be produced in this way are about two inches in diameter and six inches long. Thus, in silicon device factories, devices are formed on thin wafers cut from such ingots in a direction perpendicular to the long axis, like slices of sausage. Neither the size nor shape are conducive to good yield of devices in large production runs of transistors, integrated circuits or other 'smallsignal devices'.

For the simpler solar cell diode, area is the dominant factor. Indeed, a square foot would be a good area for a single cell; such an area just cannot be obtained at the moment, even by the expensive method of cutting ingots along the long axis, as in sawing a plank. Instead, an array of little cells has to be wired together, which greatly increases the cost and lowers the reliability of solar panels.

For these reasons, crystal growers have for a long time been exploring ways of making silicon crystals of larger area which might eliminate wasteful and arduous sawing and polishing. One technique which has recently raised hopes could be regarded as two-dimensional pulling of crystals. Crystallisation proceeds along a thin film of molten silicon lying in a mould. The method can be turned into a flow process if the film can be generated continuously. A ribbon, of theoretically unlimited length could then be produced. Tyco Laboratories, of Boston, have been exploring this process, termed edge-defined film-fed growth (EFG). As yet, few of the ribbons produced are single-crystal; most consist of large polycrystals which themselves contain imperfections in the form of dense tangles of dislocations. Nevertheless, there is often a dominance of one crystal orientation at the surface. It is thus possible that, under the right conditions, one might be able to promote totally single-crystal growth in a layer of silicon deposited on to the ribbon by one of the known epitaxial growth techniques.

Now one epitaxial growth technique is already widely used to improve the properties of integrated circuits formed on ingot-grown silicon wafers. This is the so-called chemical vapour deposition method. The layers grown are several micrometres thick and are of excellent single-crystal quality. The advantage of this deposition method is that only flowing gases are involved and the method, again a flow process, can probably be scaled up to cover large areas.

Thus, the process for making large, though imperfect silicon wafers in one step can possibly be married to this already widespread epitaxial growth technique to form a production method which yields large ribbons of crystalline silicon (it should be noted here that, for most active semiconductor devices, all of the electronic functions occur in a layer a few micrometres thick at one surface only).

Henry Kressel and co-workers at RCA Laboratories are the first to report this marriage. In Applied Physics Letters $(25,197 ;$ 1974) they note the happy result that, when the epitaxial growth from the vapour is carried out on ribbons of p-type silicon, made by Tyco Laboratories using the EFG technique, the defects in the original wafer are not strongly propagated into the newly forming crystalline lattice. This is shown clearly in $X$-ray topographs but more to the point is the finding that $p-n$ diodes with good minonity-carrier lifetime, good breakdown voltages and low leakage currents can be made by putting down first p-type and then n-type layers on to this silicon ribbon. Although the ribbon used by the investigators were only $1.3 \mathrm{~cm}$ wide, there is no basic bar to scaling this up by an order of magnitude. Ribbbon lengths of 2 metres, and widths of $2.5 \mathrm{~cm}$ have been reported unofficially.

For the integrated-cincuit and transistor manufacturer, the availability of such ribbons could give the era of single-crystal devices a new lease of life, maintaining the price-competitiveness of this form against the rapidly developing and currently less expensive thin-film device fabrication methods.

For the solar array designers, the prospect of cheaper, more reliable solar panels is improved if the ribbon method succeeds. Indeed, it is probably no coincidence that the work at RCA took place under the supervision of Paul Rappaport, one of the pioneers of the silicon photovoltaic device and coauthor of a recent survey for the National Academy of Sciences on the terrestrial use of solar converters. The latest news over the agency wires is that, despite some remaining problems with crystallinity, preparations are being made to make ribbon silicon on a factory scale.

\section{Chromosomal proteins and control of transcription}

\author{
from a Correspondent
}

The Florida Colloquium on Molecular Biology "Chromosomal Proteins and Their Role in the Regulation of Gene Expression" was held at the University of Florida in Gainesville on March 13 and 14.

Although there have been many reports correlating changes in nonhistone chromatin proteins with alterations in gene expression, there has to date been little direct evidence linking the two processes. Encouraging however were reports from two laboratories of direct demonstrations that nonhistone chromatin proteins mediate control of transcription of the histone and globin genes. G. S. Stein (University of Florida, Gainesville) has synthesised a DNA complementary to histone mRNAs and used it to establish that the transcription of histone genes from chromatin is restricted to the $\mathrm{S}$ phase of the cell cycle. Chromatin re- 